\title{
Non-Linear Multivariable Predictive Control of an Alcoholic Fermentation Process Using Functional Link Networks
}

\author{
Luiz Augusto da Cruz Meleiro ${ }^{1 *}$, Aline Carvalho da Costa $^{2}$ and Rubens Maciel Filho ${ }^{2}$ \\ ${ }^{1}$ Departamento de Engenharia Química; Universidade Federal do Paraná; C. P. 19011; luiz.meleiro@ufpr.br; \\ 81531-990; Curitiba - PR - Brazil. ${ }^{2}$ Faculdade de Engenharia Química; Universidade Estadual de Campinas; C. P. \\ 6066; 13083-970; Campinas - SP - Brasil
}

\begin{abstract}
In this work a MIMO non-linear predictive controller was developed for an extractive alcoholic fermentation process. The internal model of the controller was represented by two MISO Functional Link Networks (FLNs), identified using simulated data generated from a deterministic mathematical model whose kinetic parameters were determined experimentally. The FLN structure presents as advantages fast training and guaranteed convergence, since the estimation of the weights is a linear optimization problem. Besides, the elimination of non-significant weights generates parsimonious models, which allows for fast execution in an MPC-based algorithm. The proposed algorithm showed good potential in identification and control of non-linear processes.
\end{abstract}

Key words: Extractive alcoholic fermentation, functional link neural networks, non-linear predictive control

\section{INTRODUCTION}

Despite many advantages of using ethanol as fuel, it can substitute petroleum fuels only if its production is economically attractive. Thus, there is a great interest in the optimization of all the steps of the ethanol production process. One of the options to improve the productivity of ethanol production is the continuous extraction of ethanol. Several schemes combining fermentation with a separation process have been developed (Costa et al., 2001). Silva et al. (1999) have shown that a scheme combining a fermentor with a vacuum flash vessel presents several positive features and better performance than an industrial conventional process (Andrietta and Maugeri, 1994).

Another important aspect to be considered in the optimization of the alcoholic fermentation process is the development of an efficient control strategy, since it can minimize costs and environmental impact by maintaining the process under optimum conditions. However, biotechnological processes are characterized by their complex dynamics and modeling and control of those systems presents problems that have not yet been totally solved (Meleiro et al., 2001).

Model Predictive Control (MPC) algorithms have been widely used in industrial processes in recent years. These algorithms are well suited for high performance control of constrained multivariable processes because explicit pairing of input and output variables is not required and constraints can be incorporated directly into the controller design (Henson, 1998). On the other hand, most of the industrial applications use linear dynamic models, which may not be appropriate for highly nonlinear systems.

\footnotetext{
*Author for correspondence
} 
Although the use of non-linear models may improve the control algorithm performance, the development of such models is not always an easy task. In recent years, there has been a strong interest in the use of neural networks to describe chemical processes, due to their ability to approximate highly non-linear systems. There are several neural network structures cited in the literature, but there are no methods proposed to define the best structure to be used for a given case. In the majority of the applications the feedforward neural network (FNN) is used. A structure that has not been much explored is the functional link network (FLN). This network has been shown to have a good non-linear approximation capability, although the estimation of its weights is linear. Due to the linear estimation, its training is rapid, requires low computational effort and the convergence is guaranteed (Costa et al., 1999).

The objective of this work was to develop a nonlinear Multiple Input Multiple Output (MIMO) predictive control algorithm to control the process mentioned above, considering constraints on manipulated and controlled variables. Two
Multiple Input Single Output (MISO) Functional Link Networks (FLN) were used to identify the dynamics of the controlled variables and then used as internal models of the controller. The proposed control algorithm used the Successive Quadratic Programming method (SQP) to solve the optimization problem at each sample interval.

\section{MATERIALS AND METHODS}

\section{Extractive Alcoholic Fermentation Process}

A general scheme of the extractive alcoholic fermentation proposed by Silva et al. (1999) is shown in Fig. 1. The process consists of four interlinked units: fermentor (ethanol production unit), centrifuge (cell separation unit), cell treatment unit and vacuum flash vessel (ethanolwater separation unit). This scheme simulated industrial conditions (Andrietta and Maugeri, 1994), with the difference that only one fermentor was used instead of a cascade system, besides the flash was used to extract part of the ethanol. The substrate used was sugar-cane molasses.

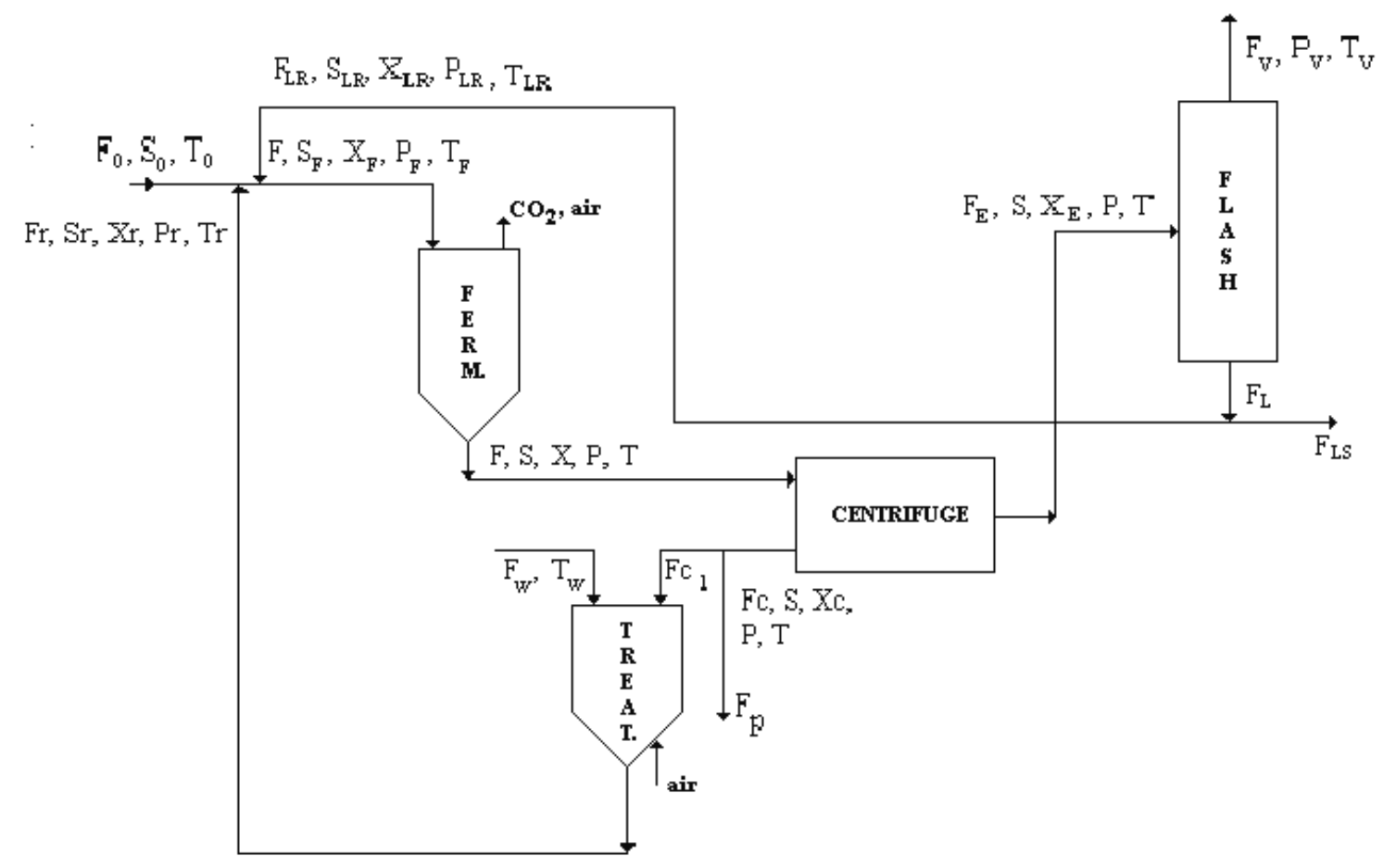

Figure 1 - Extractive Alcoholic Fermentation. 
The mathematical model used to simulate the process was experimentally validated (Costa et al., 2001).

The objective of this work was to control ethanol (P) and substrate (S) concentrations in the fermentor. Based on the dynamic behavior study performed by Costa et al. (2001), the manipulated variables must be the inlet flow rate (F0) and flash recycle rate $(r)$.

\section{Process Identification Using Functional Link Networks}

In this work, functional link neural networks were used as internal models of the predictive controller. In these networks, a non-linear functional expansion of the network inputs was initially performed and the resulting terms were combined linearly. The structure obtained had a good non-linear approximation capability, and the estimation of network weights was a linear optimization problem.

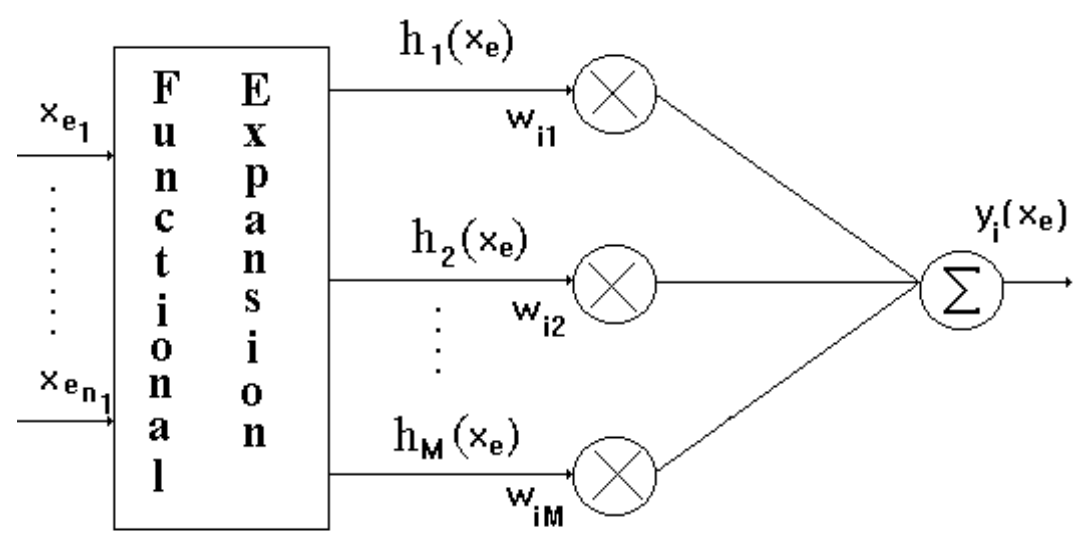

Figure 2 - General structure of a functional link network.

The general structure of an FLN is shown in Fig. 2 , where $\mathbf{x}_{\mathbf{e}}$ is the input vector and $\mathrm{y}_{\mathrm{i}}\left(\mathbf{x}_{\mathbf{e}}\right)$ is an output. The hidden layer performs a functional expansion on the input data, which maps the input space of dimension $\mathrm{n}_{1}$ onto a new space of increased dimension, $M\left(M>n_{1}\right)$. The output layer consists of $\mathrm{m}$ nodes, each one, in fact, a linear combiner. The input-output relationship of the FLN is:

$y_{i}\left(\boldsymbol{x}_{\boldsymbol{e}}\right)=\sum_{j=1}^{M} w_{i j} h_{j}\left(\boldsymbol{x}_{\boldsymbol{e}}\right), \quad 1 \leq \mathrm{i} \leq \mathrm{m}$

The most used functional expansion is the polynomial expansion. In this case, the expansion results, $\mathrm{h}_{\mathrm{j}}\left(\mathbf{x}_{\mathbf{e}}\right)$, are a series of monomials of $\mathbf{x}_{\mathbf{e}}$.

Henrique (1999) proposed a modification on the structure of the FLNs, where the output given by equation 1 was transformed by an invertible nonlinear activation function. The new output is
$y_{i}\left(\boldsymbol{x}_{\boldsymbol{e}}\right)=f_{i}\left(\sum_{j=1}^{M} w_{i j} h_{j}\left(\boldsymbol{x}_{\boldsymbol{e}}\right)\right), 1 \leq \mathrm{i} \leq \mathrm{m}$

where $f_{i}$ is an invertible non-linear function such as, for example, the sigmoidal function. This modification was made to increase the non-linear approximation ability of the FLNs. The training of the network was performed using a transformed output: the original output transformed by the inverse of the activation function $\mathrm{f}$.

Another modification was made in the FLNs to increase their non-linear approximation capability. Before the functional expansion is performed, the network inputs, $\mathbf{x}_{\mathrm{e}}$, are transformed into a greater number, $\mathrm{n}_{\mathrm{z}}$, of auxiliary inputs, $\mathbf{z}$. These auxiliary inputs are non-linear expressions of the real inputs (Costa et al., 1999). A polynomial expansion was then performed on the new inputs. The generated monomials can be found in Costa et al. (1999).

Once the monomials are generated, the network weights, $\mathrm{w}_{\mathrm{ij}}$, are estimated using an orthogonal least-squares estimator (Billings et al., 1989). Henrique (1999) also proposed that the monomials that are not significant in explaining the output 
variance can be eliminated using a method proposed by Billings et al. (1989). This procedure reduces the size and complexity of the neural network and avoids overfitting of the training data. The performance of the FLN was measured by (Milton e Arnold, 1990):

$$
\operatorname{cor}=\left(1-\frac{S E E}{S \tau \tau}\right) 100 \%
$$

where:

$S E E=\sum_{k=1}^{N}\left(y_{e}(k)-y(k)\right)^{2}, \quad S E E=\sum_{k=1}^{N}\left(y_{e}(k)-\bar{y}_{e}\right)^{2}$, $\mathrm{y}_{\mathrm{e}}(\mathrm{k})$ is the experimental output, $\mathrm{y}(\mathrm{k})$ is the network output, $\overline{y_{e}}$ is the mean value of the experimental outputs and $\mathrm{N}$ is the number of training data.

\section{Non-Linear Mimo Predictive Control}

The objective of the controller is to select a set of future control actions in order to minimize the following objective function:

$$
\begin{array}{r}
J=\sum_{j=1}^{N O} \sum_{i=1}^{N P}\left(\hat{y}_{j, k+i}^{c}-y s p_{j, k+i}\right)^{2}+ \\
\sum_{j=1}^{N I} \lambda_{j} \sum_{i=1}^{N C}\left(\Delta u_{j, k+i-1}\right)^{2}
\end{array}
$$

where $\widehat{y}^{c}$ are the controlled variables predicted by the neural networks, corrected according to equation 10; ysp are the set-point values; $\lambda$ are the weighting factors (tuning parameters that penalize the control actions); $\Delta \mathrm{u}$ are the increments on the manipulated variables; $\mathrm{NO}$ is the number of controlled variables; NI is the number of manipulated variables; NP is the prediction horizon and $\mathrm{NC}$ is the control horizon.

The objective function is subject to the following constraints:

$$
\begin{gathered}
\boldsymbol{y}_{\min } \leq \widehat{\boldsymbol{y}}_{k+i} \leq \boldsymbol{y}_{\max } \quad(i=1, \cdots, N P) \\
\boldsymbol{u}_{\min } \leq \boldsymbol{u}_{k+i-1} \leq \boldsymbol{u}_{\max } \quad(i=1, \cdots, N C) \\
\left|\boldsymbol{u}_{k+i}-\boldsymbol{u}_{k+i-1}\right| \leq \Delta \boldsymbol{u}_{\max } \quad(i=1, \cdots, N C)
\end{gathered}
$$

where $\mathbf{y}_{\min }$ and $\mathbf{y}_{\max } ; \mathbf{u}_{\min }$ and $\mathbf{u}_{\max } ; \Delta \mathbf{u}_{\min }$ and $\Delta \mathbf{u}_{\max }$ are lower and upper bounds for the vectors $\mathbf{y}, \mathbf{u}$ and $\Delta \mathbf{u}$, respectively.

The optimization algorithm calculates the values of $\Delta \mathbf{u}$ to minimize the objective function (equation 4). The future control actions are calculated over a control horizon $\mathrm{NC}$ and are kept constant for NC $\leq$ $\mathrm{i} \leq \mathrm{NP}(\mathrm{NC} \leq \mathrm{NP})$ :

$\mathbf{u}_{\mathrm{k+i}}=\mathbf{u}_{\mathrm{k+i}-1}+\Delta \mathbf{u}_{\mathrm{k+i}},(\mathrm{i}=1, \cdots, \mathrm{NC})$

$\mathbf{u}_{\mathrm{k}+\mathrm{i}}=\mathbf{u}_{(\mathrm{k}+\mathrm{NC})},(\mathrm{i}=\mathrm{NC}+1, \cdots, \mathrm{NP})$

The predictions of the neural networks are corrected by a process/model error:

$\boldsymbol{d}_{k}=\boldsymbol{y}_{k}-\hat{\boldsymbol{y}}_{k}$
$\hat{\boldsymbol{y}}_{k+i}^{c}=\widehat{\boldsymbol{y}}_{k+i}+\boldsymbol{d}_{k} \quad(i=1, \cdots, N P)$

where $\mathbf{y}_{\mathbf{k}}$ is the vector of measured outputs at the present sampling time, $\widehat{y}_{k}$ is the vector of the networks predictions (calculated at the previous sampling time) and $\widehat{\boldsymbol{y}}_{k+i}^{c}$ is the vector of the corrected networks predictions.

As the neural networks are trained to predict the controlled variables only one step ahead, in the control algorithm the networks are iterated to obtain a total of NP future predictions by using the outputs of the neural networks as their own inputs in the next iteration.

Although the optimization is based on a control horizon, only the first control action (for each manipulated variable) is implemented in the process and the optimization problem is solved again at the next sampling time. Successive Quadratic Programming (SQP), using the routine DNCONF of the IMSL math library of FORTRAN, was used to solve the optimization problem.

\section{RESULTS AND DISCUSSION}

Two MISO FLNs were used as internal models for the control algorithm. The first FLN was used to predict substrate concentration in the fermentor one step ahead with the following structure (Zhan and Ishida, 1997; Santos et al., 2000): 
- Inputs: $\quad \mathrm{S}_{\mathrm{k}}, \mathrm{F}_{0 \mathrm{k}}$ and $\mathrm{r}_{\mathrm{k}}$

- Output: $S_{k+1}$

The second FLN was used to predict ethanol concentration in the fermentor one step ahead with the following structure:

- Inputs: $\quad \mathrm{P}_{\mathrm{k}}, \mathrm{F}_{0 \mathrm{k}}$ and $\mathrm{r}_{\mathrm{k}}$

- Output: $\mathrm{P}_{\mathrm{k}+1}$

where $\mathrm{k}$ is the present sampling time.

The training data were obtained by performing random step changes in the manipulated variables: inlet flow rate, $\mathrm{F}_{0}$, and flash recycle rate, $r$. The inputs were transformed into auxiliary inputs for both FLNs. After some tests the auxiliary input vectors that led to the best results were chosen:

$$
\boldsymbol{z}_{1}^{\mathrm{T}}=\left[\begin{array}{lll}
F_{0 k} & r_{k} & \frac{1}{S_{k}}
\end{array}\right]
$$

$$
\boldsymbol{z}_{2}^{\mathrm{T}}=\left[\begin{array}{lll}
F_{0 k} & r_{k} & \frac{1}{P_{k}}
\end{array}\right]
$$

Tests were made to determine the functional expansion degree and activation function that led to the best training performance of the networks (Costa et al., 1999). For both FLNs the functional expansion degree chosen was 6 and the activation function was:

$$
f\left(\sum_{j=1}^{M} w_{i j} h_{j}\left(\boldsymbol{x}_{\boldsymbol{e}}\right)\right)=\frac{1}{\sum_{j=1}^{M} w_{i j} h_{j}\left(\boldsymbol{x}_{\boldsymbol{e}}\right)}
$$

Many authors suggested that the neural networks used as internal models of MPC schemes should be validated by testing their capacity to predict steady states of the process (Zhan and Ishida, 1997; Santos et al., 2000). Figs. 3-6 show the results when the trained FLNs were used to predict the steady states of the extractive alcoholic fermentation process for different values of the manipulated variables $\left(\mathrm{F}_{0}\right.$ and $\left.\mathrm{r}\right)$. The trained networks showed good performance.

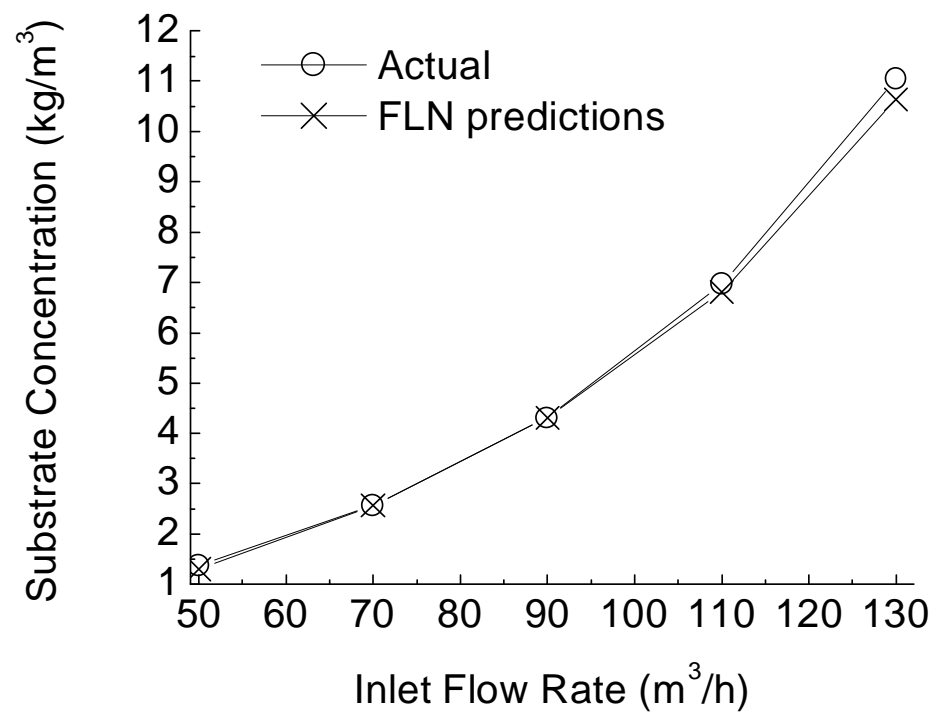

Figure 3 - Performance of the FLN to predict substrate concentration steady states for different values of the inlet flow rate. 


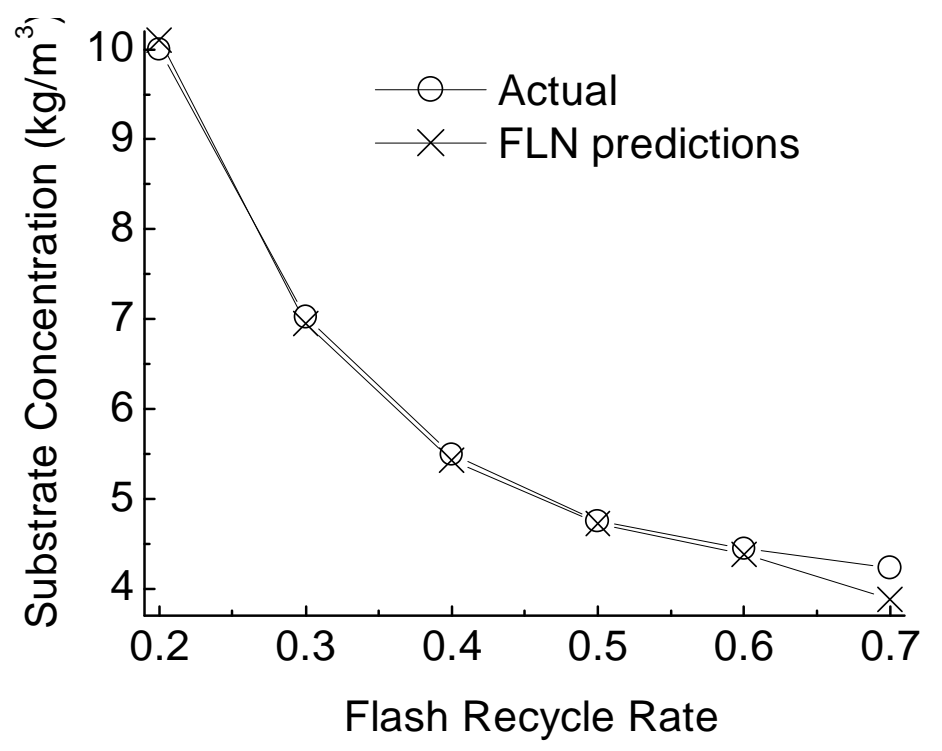

Figure 4 - Performance of the FLN to predict substrate concentration steady states for different values of the flash recycle rate.

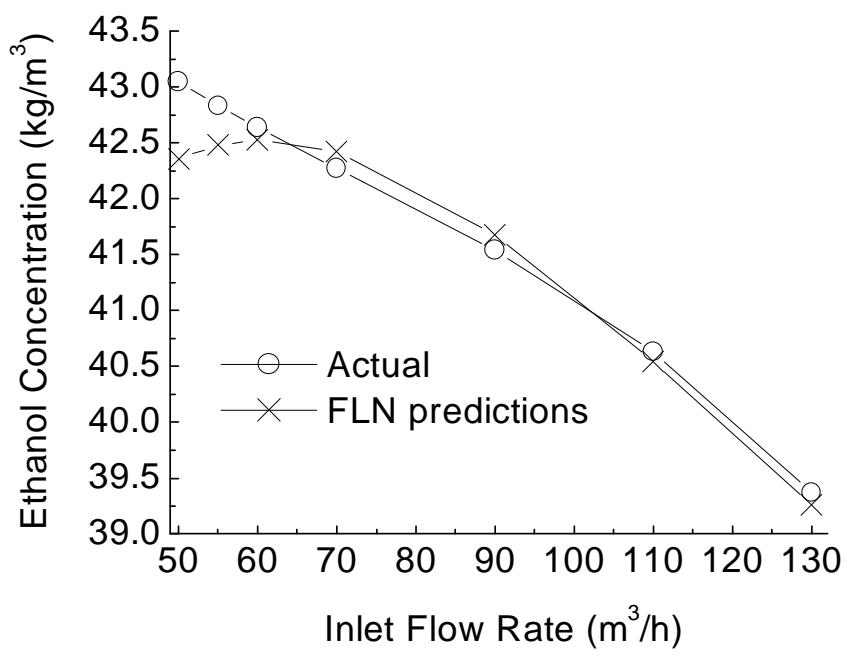

Figure 5 - Performance of the FLN to predict product concentration steady states for different values of the inlet flow rate. 


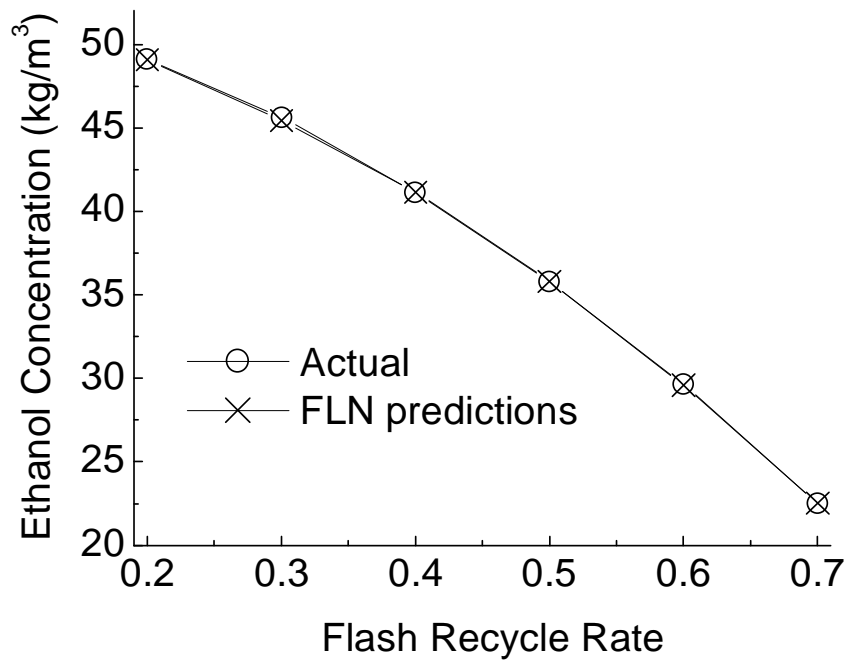

Figure 6 - Performance of the FLN to predict product concentration steady states for different values of the flash recycle rate.
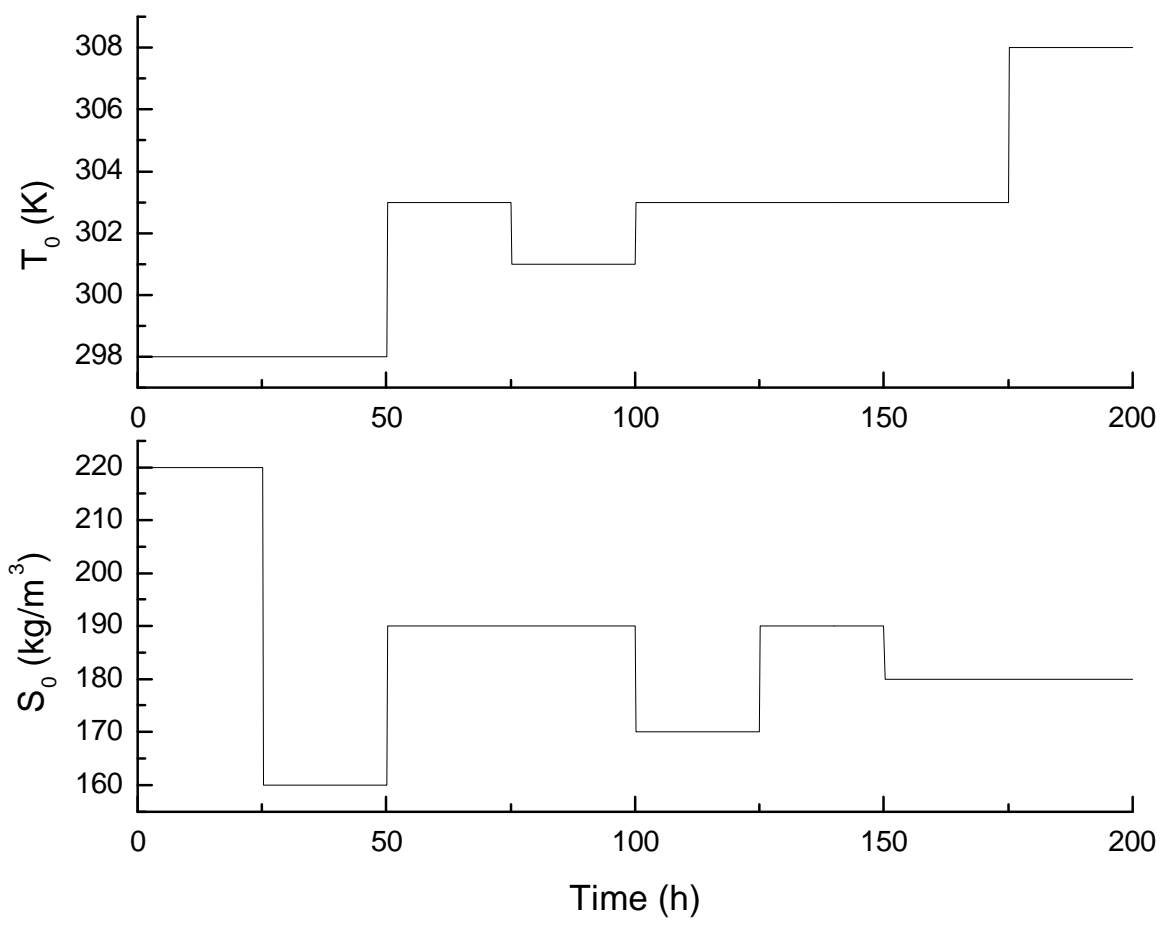

Figure 7 - Disturbances imposed on S0 and T0. 


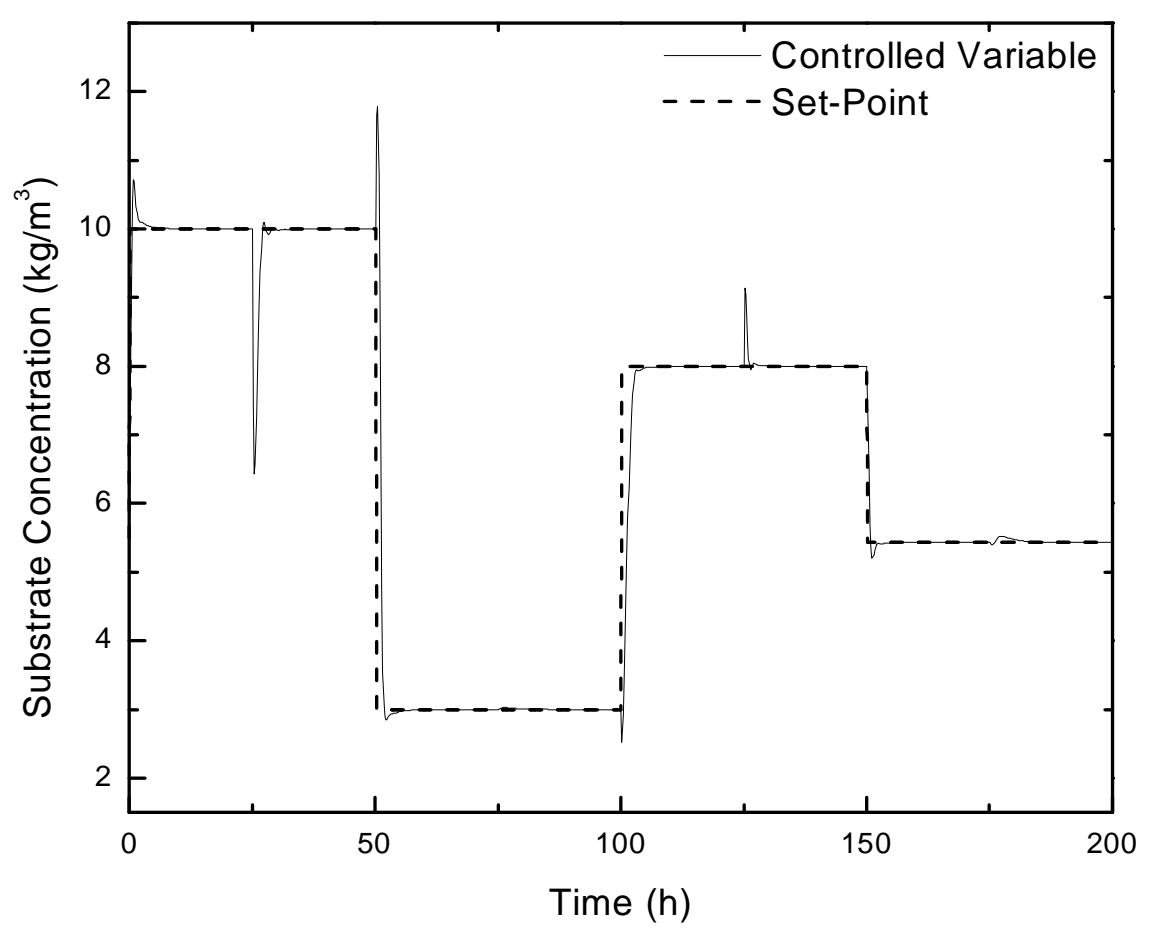

Figure 8 - Servo and regulatory control for substrate concentration.

The FLNs described above were used as internal models of the predictive controller. The sampling time was chosen as 12 minutes, considering that an HPLC (High Performance Liquid Chromatograph) was used to measure substrate and ethanol concentrations on-line (Andrietta, 1994). The controlled and manipulated variables constraints were as follows:

$$
\begin{aligned}
& S_{k+i} \geq 0 \quad(i=1, \cdots, N P) \\
& 60 \leq F_{0 k+i-1} \leq 150 \quad(i=1, \cdots, N C) \\
& 0.2 \leq r_{k+i-1} \leq 0.7 \quad(i=1, \cdots, N C) \\
& \left|F_{0 k+i}-F_{0 k+i-1}\right| \leq 50 \quad(i=1, \cdots, N C) \\
& \left|r_{k+i}-r_{k+i-1}\right| \leq 0.1 \quad(i=1, \cdots, N C)
\end{aligned}
$$

and the controller parameters were chosen after many tests: $\mathrm{NP}=8, \mathrm{NC}=3$ and $\lambda^{\mathrm{T}}=\left[\begin{array}{ll}0.01 & 70] \text {. The }\end{array}\right.$ performance of the controller was tested by changing simultaneously the set-points and disturbance variables that influence the controlled variables. The main disturbance variables in this process are the inlet substrate concentration, $S_{0}$, and the inlet temperature, $\mathrm{T}_{0}$. The disturbances made in $S_{0}$ and $T_{0}$ are shown in Fig. 7. The setpoint values for these variables were $180 \mathrm{~kg} / \mathrm{m} 3$ and $303 \mathrm{~K}$, respectively.

Fig. 8 shows the performance of the controller when set-point changes in substrate concentration were performed simultaneously with the disturbances shown in Fig. 7. Note that the controller maintained the output concentration at the desired value.

Fig. 9 illustrates the corresponding behavior of product concentration. It can be noticed that the controller was able to reject the disturbances that affected this variable. Fig. 10 depicts the control actions. Fig. 11 shows the results when set-point changes in product concentration were performed simultaneously with the disturbances shown in Fig. 7. Fig. 12 illustrates the behavior of substrate concentration. It can be seen that the non-linear controller presented good performance to lead the system to new set-points and to reject disturbances. Fig. 13 depicts the control actions. 


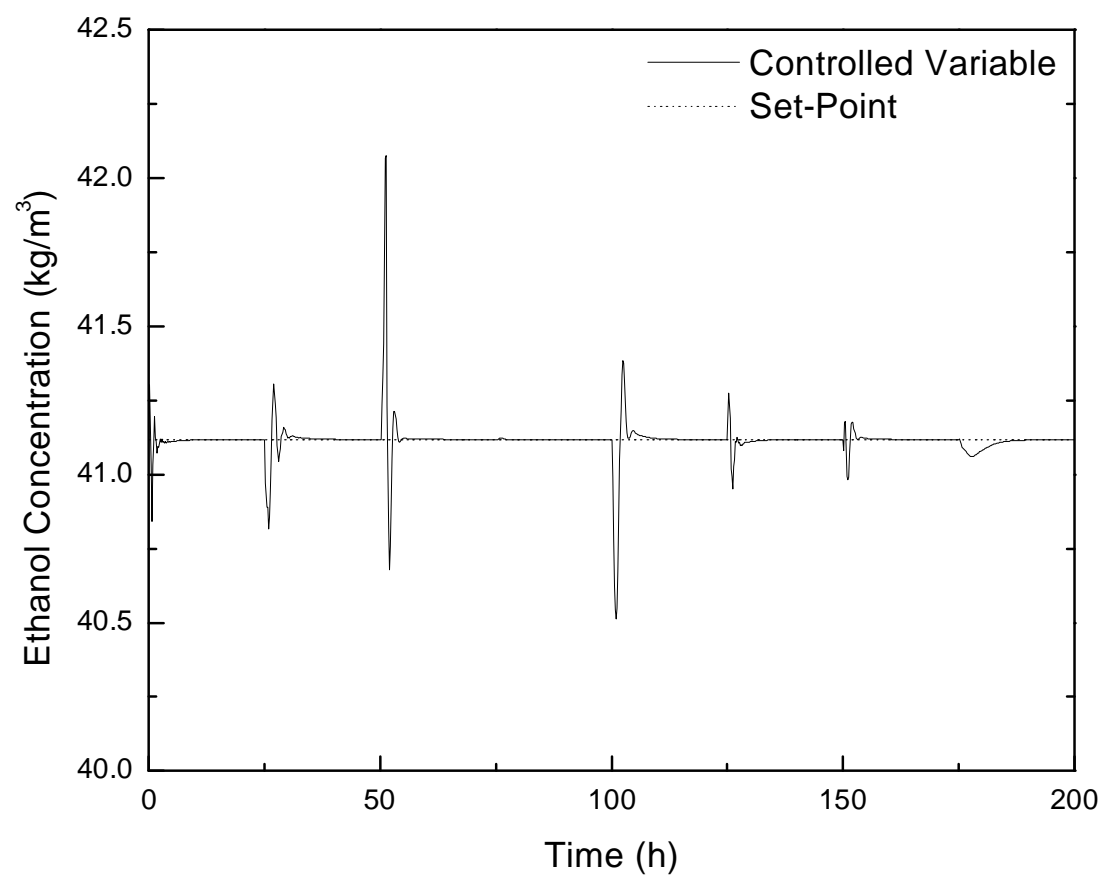

Figure 9 - Regulatory control for product concentration

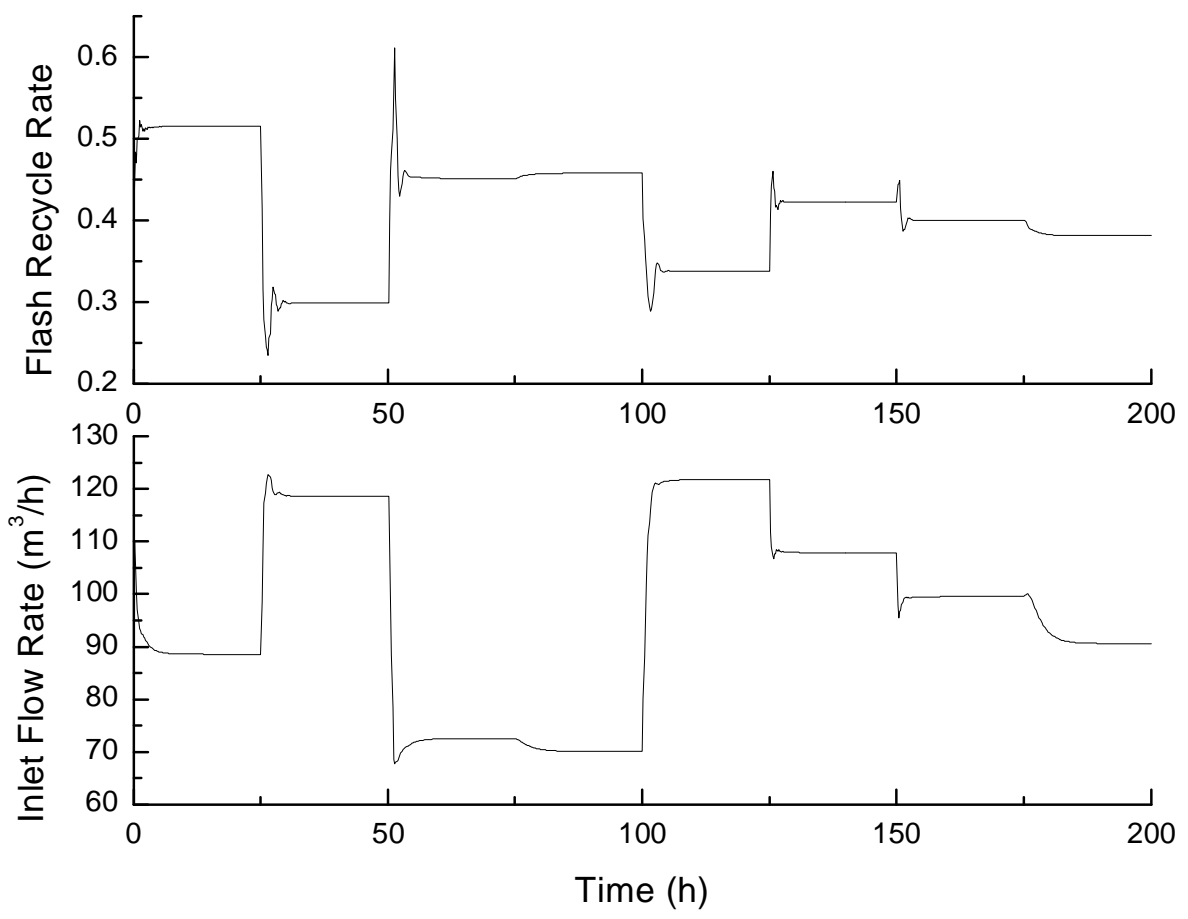

Figure 10 - Control actions related to Figures 8 and 9. 


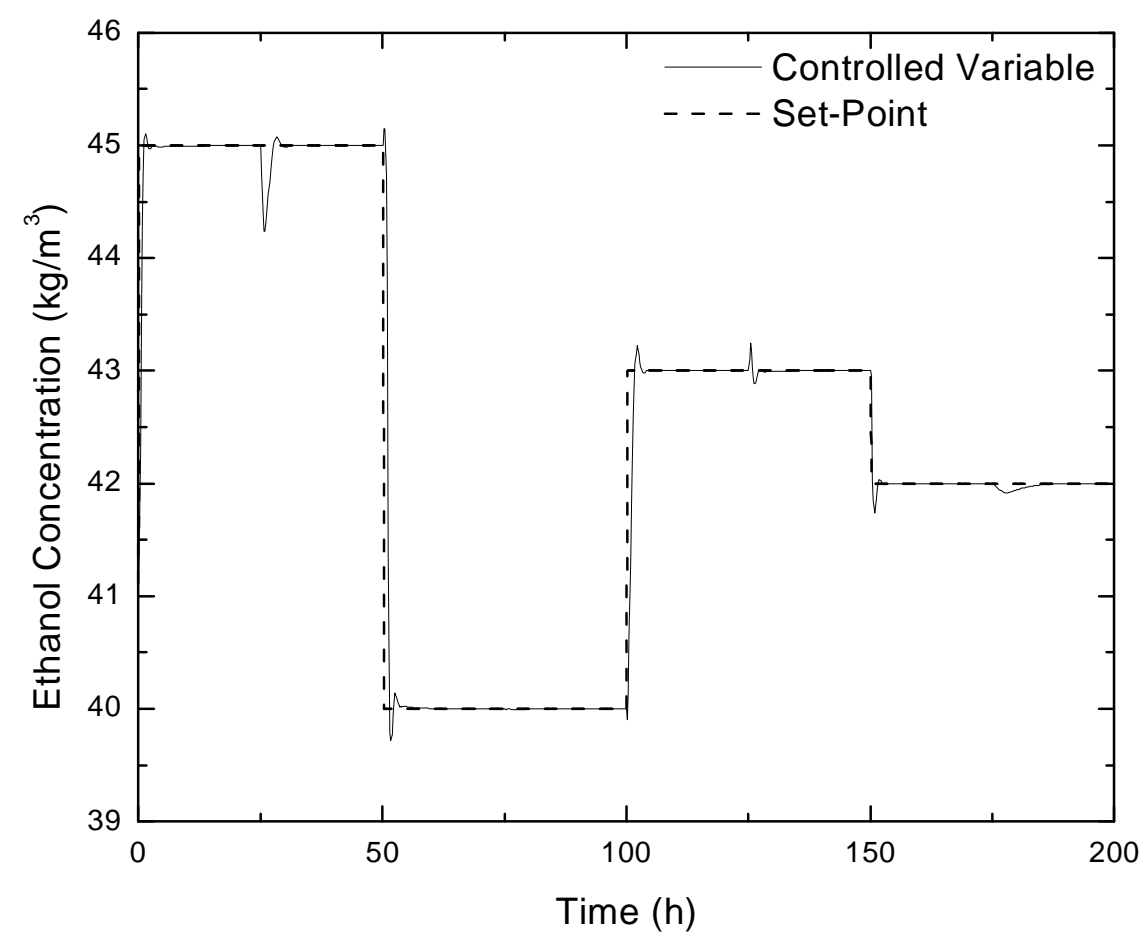

Figure 11 - Servo and regulatory control for product concentration.

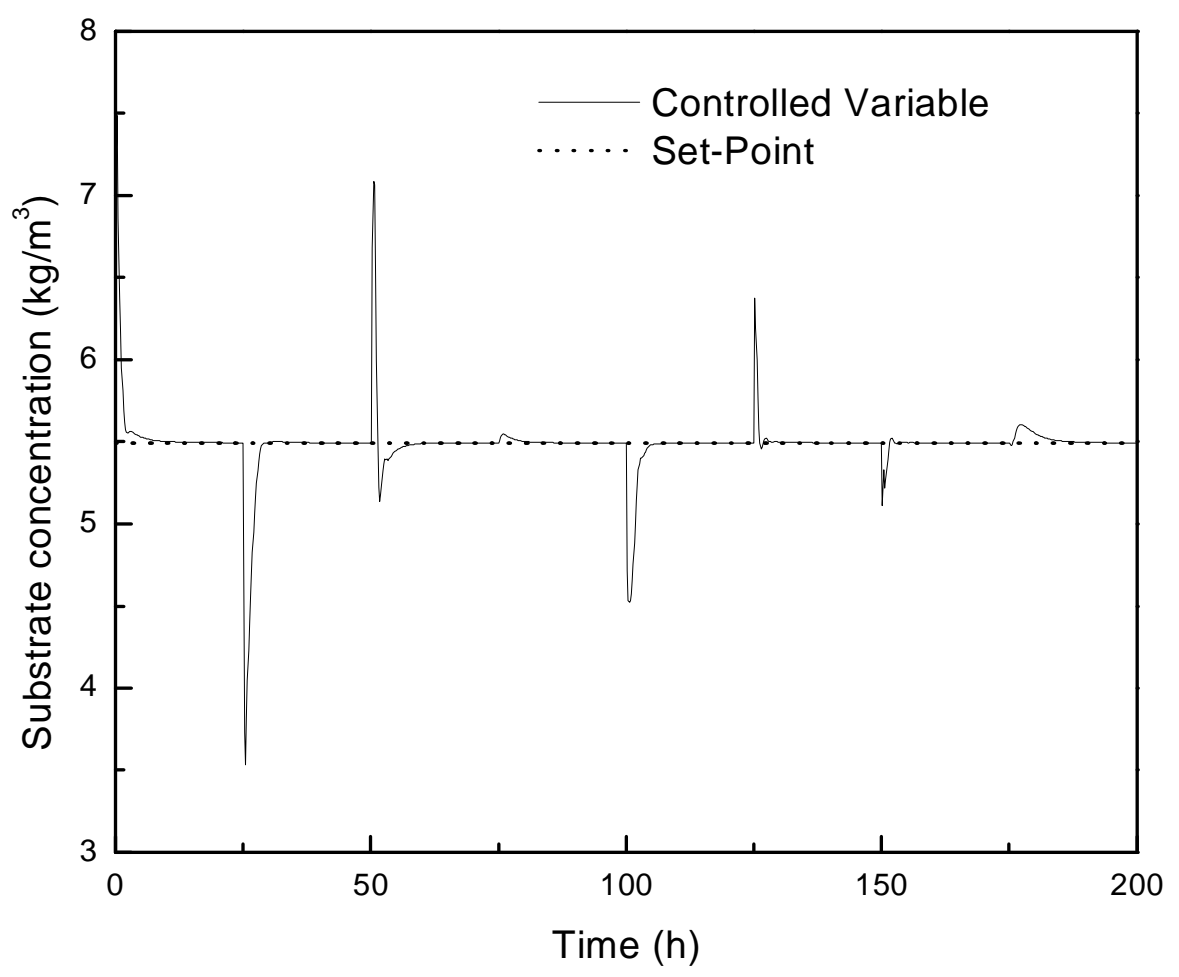

Figure 12 - Regulatory control for substrate concentration. 


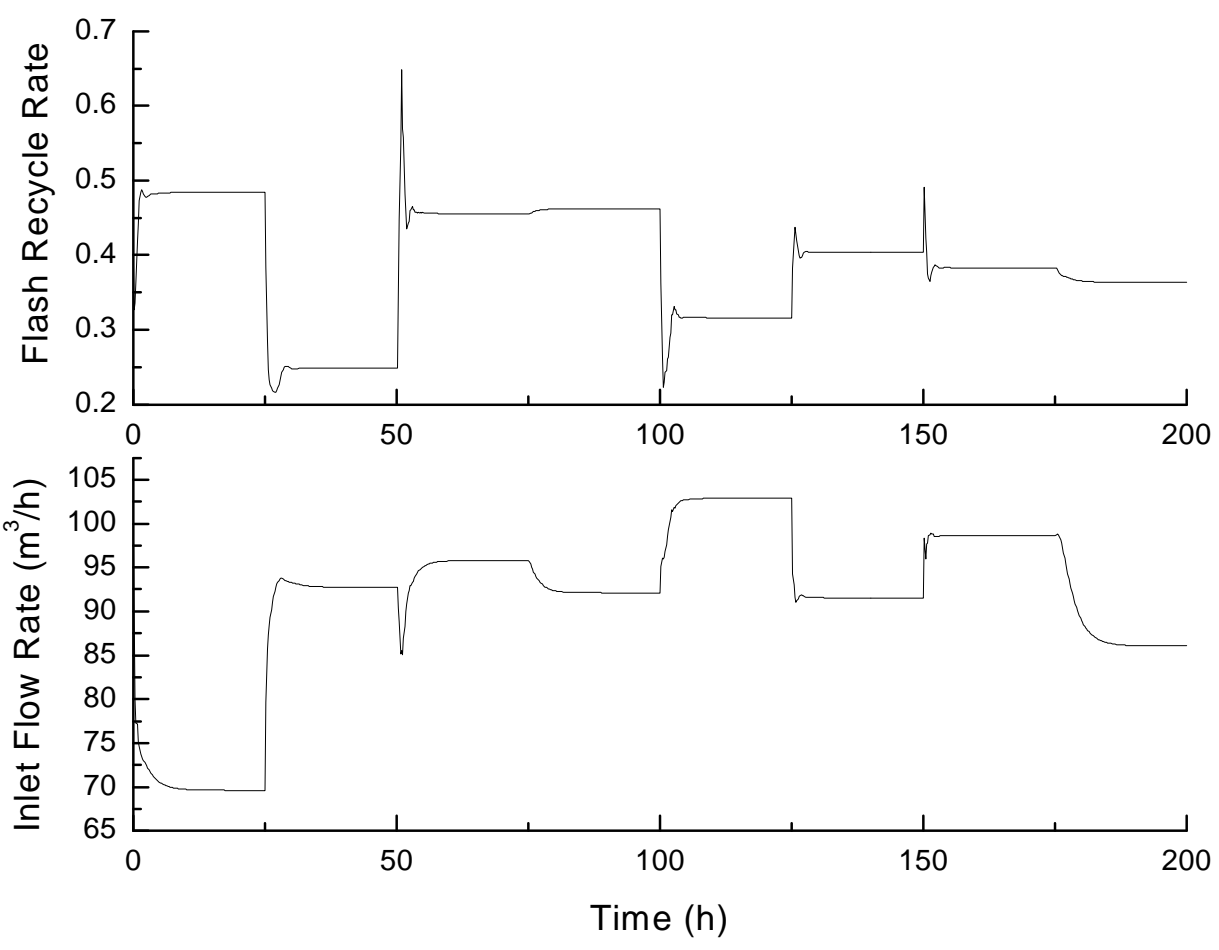

Figure 13 - Control actions related to Figs. 11 and 12.

\section{CONCLUSIONS}

In this work, two MISO Functional Link Networks (FLNs) were applied to identify an extractive alcoholic fermentation process using simulated data generated by a deterministic model whose parameters were obtained from the experiments. The FLNs represented accurately the dynamic and static behavior of the process and presented good potential to be used as internal models of a control algorithm. This structure presented the advantages of fast training and guaranteed convergence, since the estimation of the weights is a linear optimization problem. Besides, the elimination of non-significant weights generated parsimonious models, which allowed for fast execution in an MPC-based algorithm.

The non-linear MIMO predictive controller developed in this work used FLNs as internal models and was applied to control the extractive alcoholic fermentation process. By solving a constrained MIMO optimization problem over a future time horizon, this highly non-linear system could be successfully controlled. The performance of the proposed controller was evaluated for servo and regulatory problems, and in both cases it showed satisfactory results.

\section{RESUMO}

Neste trabalho um controlador preditivo não linear multivariável foi desenvolvido para um processo de fermentação alcoólica extrativa. O modelo interno do controlador foi representado por duas redes do tipo Functional Link (FLN), identificadas usando dados de simulação gerados a partir de um modelo validado experimentalmente. A estrutura FLN apresenta como vantagem o treinamento rápido e convergência garantida, já que a estimação dos seus pesos é um problema de otimização linear. Além disso, a eliminação de pesos não significativos gera modelos parsimoniosos, o que permite a rápida execução em algoritmos de controle preditivo baseado em modelo. Os resultados mostram que o algoritmo proposto tem grande potencial para identificação e controle de processos não lineares. 


\section{REFERENCES}

Andrietta, S. R. and Maugeri, F. (1994), Optimum Design of a Continuous Fermentation Unit of an Industrial Plant for Alcohol Production, In Adv. Bioprocess Engng., 47-52.

Billings, S. A.; Chen, S. and Korenberg, M. J. (1989), Identification of MIMO Non-Linear Systems Using a Forward-Regression Orthogonal Estimator, Int. J. Control, 49, 2157-2189.

Costa, A. C.; Atala, D. I. P.; Maciel Filho, R. and Maugeri Filho, F. (2001), Factorial Design and Simulation for the Optimization and Determination of Control Structures for an Extractive Alcoholic Fermentation, Process Biochemistry, 37, 125-13.

Costa, A. C.; Henriques, A. W. S.; Alves, T. L. M.; Maciel Filho, R. and Lima, E. L. (1999), A Hybrid Neural Model for the Optimization of Fed-Batch Fermentations, Braz. J. Chem. Engng, 16, 53-63.

Henrique, H. M. (1999), A Contribution to the Study of Neural Networks Applied to Process Control. In Portuguese. PhD Thesis, COPPE/UFRJ, RJ, Brazil.

Henson, M. A. (1998), Nonlinear Model Predictive Control: Current Status and Future Directions, Comp. Chem. Engng., 23, 187-202.

Meleiro, L. A. C.; Maciel Filho, R.; Campello, R. J. G. B. and Amaral, W. C. (2001), Hierarchical Neural Fuzzy Models as a Tool for Process Identification: A Bioprocess Application. In: Mujtaba, I. M. and Hussain, M. A. (Eds.), Application of Neural Network and Other Learning Technologies in Process Engineering. Imperial College Press.

Milton, J. S. and Arnold, J. C. (1990), Introduction to Probability and Statistics. New York: McGraw Hill.

Santos, V. M. L.; Carvalho, F. R. and De Souza Jr., M. B. (2000), Predictive Control Based on Neural Networks: An Application to a Fluid Catalytic Cracking Industrial Unit, Braz. J. Chem. Eng., 17, 897-905.

Silva, F. L. H.; Rodrigues, M. I. and Maugeri, F. (1999), Dynamic Modelling, Simulation and Optimization of an Extractive Continuous Alcoholic Fermentation. J. Chem. Tech. Biotechnol., 74, 176-182.

Zhan, J. and Ishida, M. (1997), The Multi-Step Predictive Control of Nonlinear SISO Processes with a Neural Model Predictive Control (NMPC) Method, Comp. Chem. Engng., 21, 201-210.

Received: September 29, 2004; Revised: February 25, 2005; Accepted: March 25, 2005 